\title{
Formation of globular clusters with multiple populations
}

\author{
T. Decressin
}

INAF - Osservatorio Astronomico di Roma, via Frascati 33, 00040 Monte Porzio Catone (RM), Italy

email: tdecressin@googlemail.com

\begin{abstract}
Spectroscopic and photometric evidences have led to a complete revision of our understanding of globular clusters with the discovery of multiple stellar populations which differ chemically. Whereas some stars have a chemical composition similar to fields stars, others show large star-to-star variations in light elements ( $\mathrm{Li}$ to $\mathrm{Al}$ ) while their composition in iron and heavy elements stay constant. This peculiar chemical pattern can be explained by self-pollution of the intracluster gas occurring in the early evolution of clusters. Here the possible impact from a first generation of fast rotating stars to the early evolution of globular clusters is presented. The high rotation velocity will allow the stars to rotate at the break-up velocity and release matter enrich in H-burning which in turn will produce new stars with a chemical composition in agreement with observations. The massive stars have also an important role to clear the cluster from the remaining gas left after the star formation episodes. If the gas expulsion is fast enough, the strong change in the potential well will lead to the loss of stars occupying the outer part of the cluster. As second generation stars are preferentially born in the cluster centre, the ratio of second to first generation stars will increase over time to match the present ratio determined by observations. Considerations on the properties of low-mass stars still present in globular clusters will also be presented.
\end{abstract}

Keywords. Globular clusters, multiple population, stars: massive stars, stars: low-mass

\section{Introduction}

Over the last decades our understanding of globular clusters deeply challenged. Indeed with the determination of abundances variations in light elements by spectroscopy (see Gratton et al. 2012, for a review) and the broad or multiple main sequences and giant branches by precise photometry (Bedin et al. 2004) a new paradigm has risen. Those clusters harbour several population or generations of stars. One has similar abundance pattern as fields stars of similar metallicity while other shows signs of H-burning at high temperature (Prantzos et al. 2007) with He increase and anticorrelations between Li-Na, C-N, O-Na and Mg-Al while heavier element stay constant (Gratton et al. 2012).

Different scenario have been proposed to explain these features which involved a selfenrichment inside the clusters during its evolution by different stellar polluters: massive AGB stars (Ventura et al. 2001, 2013; D'Ercole et al. 2010, 2011, 2012), fast rotating massive stars (Maeder \& Meynet 2006; Decressin et al. 2007b; Krause et al. 2013), massive binaries stars (de Mink et al. 2009), and super-massive stars (Denissenkov \& Hartwick 2014). In the following we will focus on the role of fast rotating massive stars.

\section{Effect of massive stars on the evolution of globular clusters}

One possible candidate for the self-enrichment in globular clusters are the fast rotating massive stars. If stars more massive than $20-25 \mathrm{M}_{\odot}$ have high enough rotation rate at 


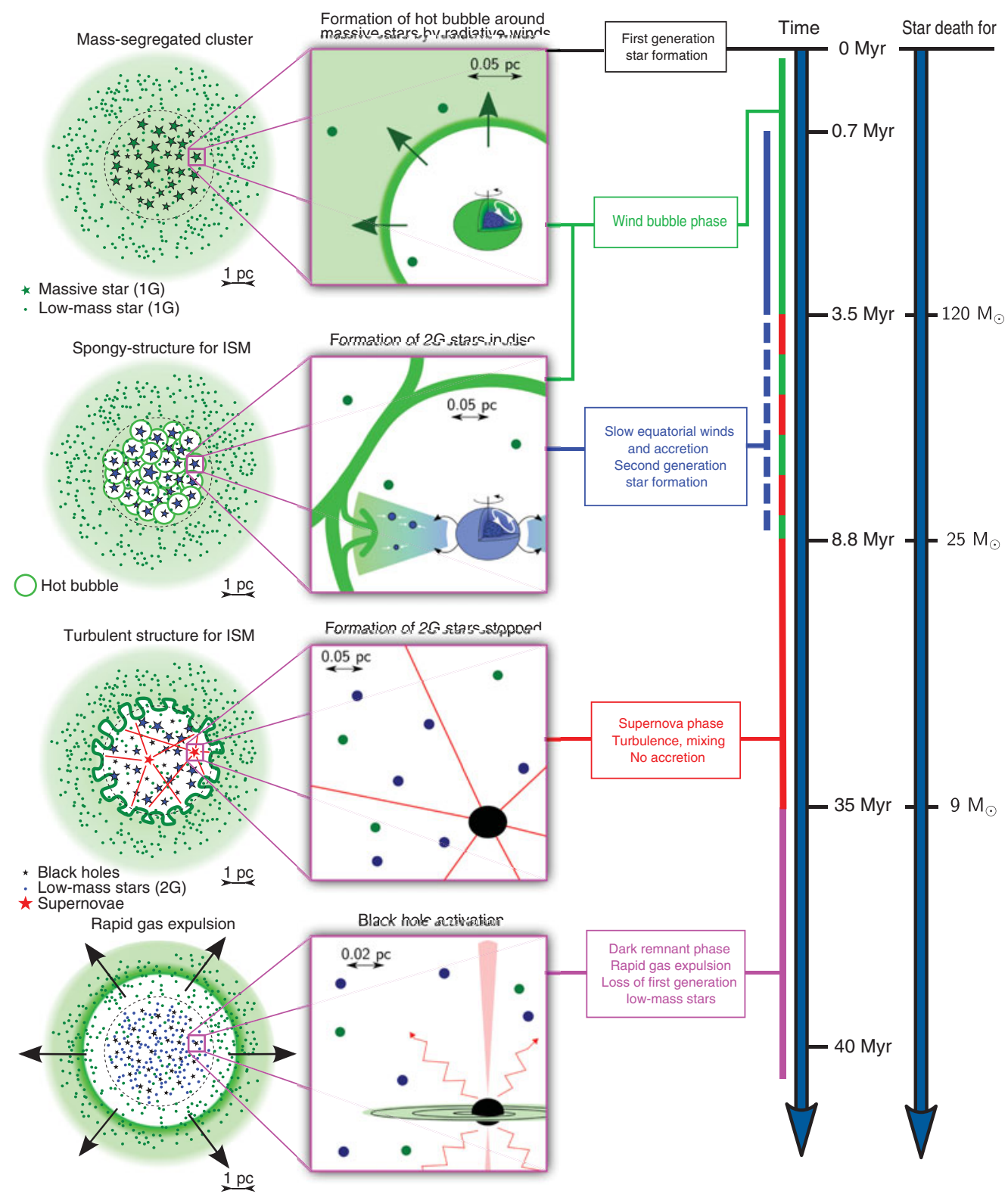

Figure 1. Sketch of the model of first $40 \mathrm{Myr}$ of evolution of a proto-globular cluster populated with fast rotating massive stars leading to two chemically-distinct population of stars as well as the loss of the outer part. A typical timescale is given on the right axis as well as the mass of the most massive star living in the cluster. The massive stars at the centre of the cluster first form hot bubble around them leading to a spongy structure of the ISM. Their fast rotation leads to the creation of a disc around them. The ISM is then been accreted into the disc and new stars will be born with a different chemical composition compared to pristine gas. Then the supernovae phase starting between 3.5 and $8.8 \mathrm{Myr}$ is not enough to inbound the gas due to Rayleigh-Taylor instability but the high turbulence of the gas inhibits further stars formation. When the supernovae cease after about $35 \mathrm{Myr}$, the ISM can be accreted by dark remnant. Then the activation of dark remnant allow the cluster to efficiently lose its gas left after star formation. 
their surface, the rotation-induced mixing will bring matter synthesised in the core to the surface and the centrifugal forces will balance the gravity at the equator so that a slow mechanical wind creates a disk around the stars. This disc will then mix with pristine matter left after the first stars formation to form new stars loaded a H-burning products similar to the observed stars in globular clusters (see Fig. 1 and Decressin et al. 2007b).

However this scenario will only produce a limited amount of about $10 \%$ of stars with abundance anomalies whereas a much higher fraction is detected in nowadays clusters (about 60\% following Carretta et al. 2010). One possibility to alleviate this constraint is to assume that the cluster was much more massive at birth (by at least a factor 2030, see Decressin et al. 2007a) and that the cluster lost a large part of its low-mass chemically-normal stars during its evolution. Dynamical studies have shown that twobody relaxation acts in too large timescales to be relevant as shown by Decressin et al. (2008) (see also D'Ercole et al. 2008).

A more efficient mechanism can be the violent gas expulsion after the stars formation. As the massive stars will seat at the centre of the cluster, the chemically-anomalous stars will also born in the centre. On the contrary the outer part of the cluster will harbour only chemically-normal stars. If the gas expulsion is fast enough (in about less than the cluster crossing time) the potential well of the cluster will be heavily affected and the stars in the outer part of the cluster will be released into the halo of the Galaxy (Decressin et al. 2010).

The crucial point to an efficient enough gas expulsion is its short timescale as the physical process responsible for it need to transfer a large amount of energy to the gas rapidly. Supernovae explosion will not be powerful enough to remove efficiently the gas as they lead to the formation of an expanding superbubble that will be destroyed by Rayleigh-Taylor instability before it reaches escape speed. This will lead to a highly turbulent ISM which will inhibit further stellar formation. A more power mechanism is required to expel the gas, which might plausibly be provided by a coherent onset of accretion onto the stellar remnants once the supernovae cease (Krause et al. 2012). However as the large fraction of the stars ejected from the cluster will populate the Galactic halo, their number will conflict with observations in Fornax (see Larsen et al. 2012).

\section{Accretion on pre main sequence stars}

One possibility to distinguish between the different proposed scenarios to explain the abundance anomalies in globular clusters is to look at the properties of the resulting low-mass stars. Recently Bastian et al. (2013) have proposed an alternative to form chemically-anomalous stars with their scenario of early disk accretion. As the timescale for the evolution of massive stars is shorter than the pre-main sequence (PMS) of lowmass stars, the accretion disk around proto low-mass stars will be replace by matter ejected by massive binaries. In this case only one generation is needed to explain the two population of stars in globular clusters. This model can help to alleviate the mass-budget problem explained previously.

As already noted by D'Antona et al. (2014) stars on the PMS will acquire a radiative core and will no longer be fully convective after a few Myr. This poses some additional constraints on the timescale during which the early accretion can take place. As the accreted matter is loaded in H-burning products and has a high helium abundance, its mean molecular weight is heavier than the stellar interior. Such condition is favourable to develop thermohaline instabilities which will mix and homogenise the stellar interior(Ulrich 1972). This mechanism has already been used to explain abundances variations along the 


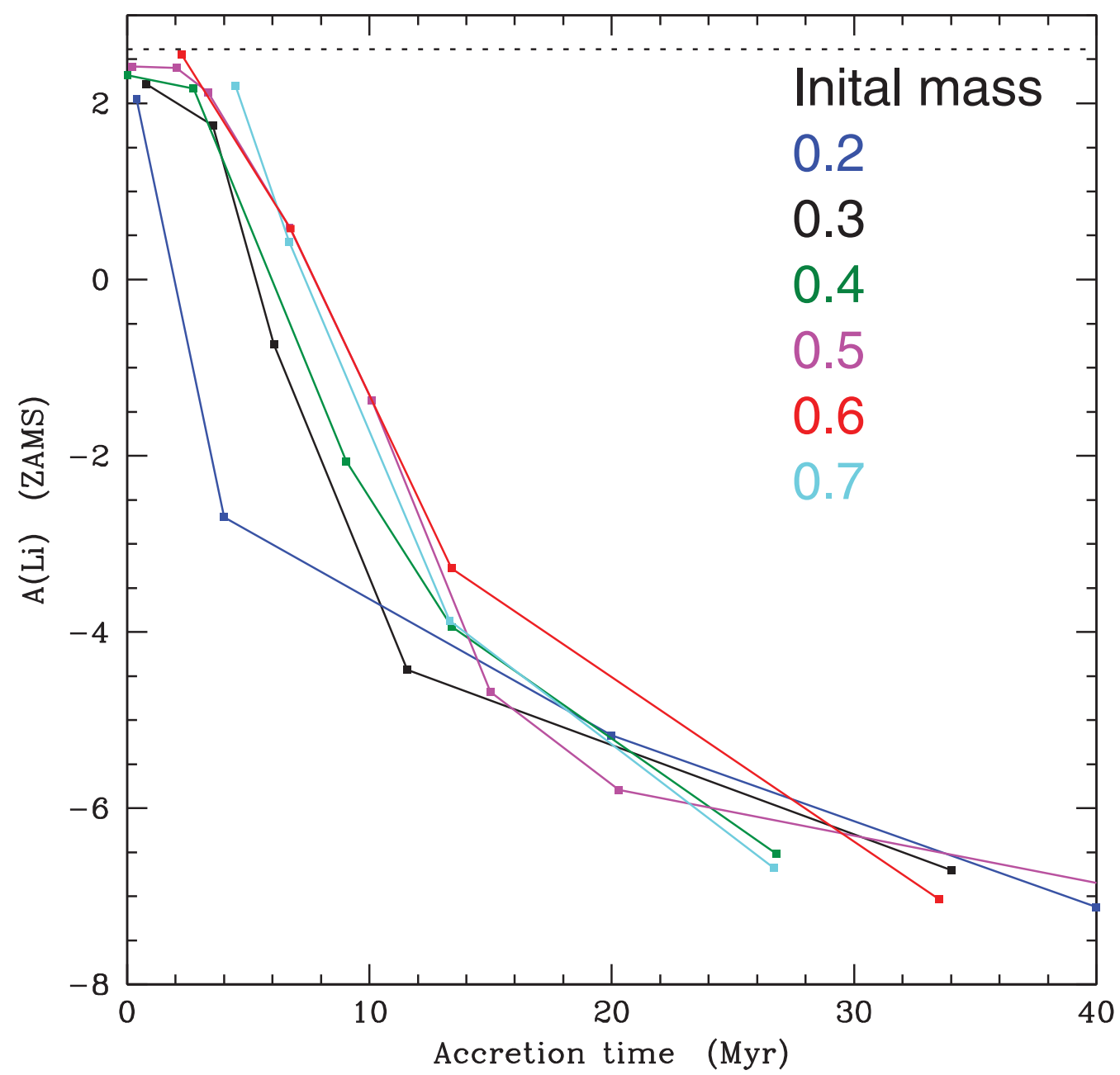

Figure 2. Abundance of $\mathrm{Li}$ at the surface of a $0.8 \mathrm{M}_{\odot}$ stars on the ZAMS as function of the timescale of the accretion for a proto-star of 0.2 to $0.7 \mathrm{M}_{\odot}$.

RGB (see e.g., Charbonnel \& Lagarde 2010). In PMS stars, fragile element as lithium will be heavily affected by this process as its burning temperature is low enough to be reached below the convective envelope. Thermohaline instabilities will then lead to the complete destruction of lithium inside the star as illustrated in Fig. 2. This is in contradiction with the observations showing that chemically anomalous stars still present lithium on their surface in the main sequence (see e.g., Lind et al. 2009; Monaco et al. 2012; Dobrovolskas et al. 2014).

\section{Horizontal branch in $\omega$ Cen}

One difference between chemically normal and anomalous low-mass stars will also be visible along the horizontal branch (HB). Indeed stars with abundance anomalies have a higher initial He abundance and hence they evolve faster on the main sequence. Assuming similar mass loss on the red giant branch the position on the HB will reflect their mass and composition: lighter stars will become hotter due to their smaller envelope (see e.g. Chantereau et al. 2015). 
In particular in $\omega$ Cen the presence of a large number of stars in the blue hook of the HB (i.e., the hottest in this cluster, see Anderson \& van der Marel 2010) has for a long time remained a puzzle. Tailo et al. (2015) have shown that this structure can be understood as the progeny of stars which had late helium-core flash ignition followed by deep mixing. However an increase of the core mass up to $0.04 \mathrm{M}_{\odot}$ is also needed to fully explain their luminosity function. This core-mass increase can be achieved by a dispersion in rotation rates by the progenitors. Indeed, low-mass stars in the dense environment of the cluster's central regions see their PMS accretion disk disrupts at an early stage leaving them with a high rotation at the ZAMS. Following Mengel \& Gross (1976) their high rotation rate will lead to higher core mass at the time of the He-flash. Rotation may also account for frequent late-flash-mixing events in massive globular clusters.

\section{The extended main-sequence turn-off cluster NGC 1856}

Finally it has recently been discovered multiple or extended turn-offs in young clusters in the Magellanic Clouds (see e.g., Mackey et al. 2008; Milone et al. 2009). Two interpretations of this feature have been proposed: a significant age spread of several $100 \mathrm{Myr}$ in clusters aged 1-2 Gyr (e.g. Milone et al. 2009; Girardi et al. 2011; Goudfrooij et al. 2011; Rubele et al. 2013) or a spread in stellar rotation (e.g. Bastian \& de Mink 2009; Niederhofer et al. 2015).

D'Antona et al. (2015) have recently focused on the young cluster NGC 1856, showing a splitting in the upper part of the main sequence observed by Milone et al. (2015). With the use of population synthesis programs available from the Geneva stellar models (Georgy et al. 2014), this cluster can be seen as the superposition of two main populations having the same age of about $350 \mathrm{Myr}$. Two third are very rapidly rotating stars while a third are slowly/non-rotating stars. Stars with rapid rotation are common among B and A stars, whereas binary synchronization is a possible process behind the slowly/nonrotating population (see Zahn 1977). One outcome of this explanation is that a large number of the slowly/non-rotating stars should still be in a loose binary system as the cluster is not dense enough to disrupt most of binaries. In such binary systems, Roche lobe overflow will occur when the primary leaves off the main sequence. In this case the primary may not be able to ignite helium in their core and the red clump is only populated by the former fast rotating population which is consistent with the observed cluster.

\section{Acknowledgement}

T.D. acknowledges support from the UE Program (FP7/2007/2013) under grant agreement number 267251 of Astronomy Fellowships in Italy (ASTROFit).

\section{References}

Anderson, J. \& van der Marel, R. P. 2010, ApJ, 710, 1032

Bastian, N. \& de Mink, S. E. 2009, MNRAS, 398, L11

Bastian, N., Lamers, H. J. G. L. M., de Mink, S. E., et al. 2013, MNRAS, 436, 2398

Bedin, L. R., Piotto, G., Anderson, J., et al. 2004, ApJ, 605, L125

Carretta, E., Bragaglia, A., Gratton, R. G., et al. 2010, A\& $A, 516$, A55

Chantereau, W., Charbonnel, C., \& Decressin, T. 2015, A\&A, 578, A117

Charbonnel, C. \& Lagarde, N. 2010, A\& A, 522, A10

D'Antona, F., Di Criscienzo, M., Decressin, T., et al. 2015, MNRAS, 453, 2637

D'Antona, F., Ventura, P., Decressin, T., Vesperini, E., \& D'Ercole, A. 2014, MNRAS, 443, 3302 
de Mink, S. E., Pols, O. R., Langer, N., \& Izzard, R. G. 2009, A\& A, 507, L1

Decressin, T., Baumgardt, H., Charbonnel, C., \& Kroupa, P. 2010, A\& $A$, 516, A73

Decressin, T., Baumgardt, H., \& Kroupa, P. 2008, A\&A, 492, 101

Decressin, T., Charbonnel, C., \& Meynet, G. 2007a, A\&A, 475, 859

Decressin, T., Meynet, G., Charbonnel, C., Prantzos, N., \& Ekström, S. 2007b, A\&A, 464, 1029

Denissenkov, P. A. \& Hartwick, F. D. A. 2014, MNRAS, 437, L21

D'Ercole, A., D'Antona, F., Carini, R., Vesperini, E., \& Ventura, P. 2012, MNRAS, 423, 1521

D'Ercole, A., D'Antona, F., Ventura, P., Vesperini, E., \& McMillan, S. L. W. 2010, MNRAS, 407,854

D'Ercole, A., D'Antona, F., \& Vesperini, E. 2011, MNRAS, 415, 1304

D'Ercole, A., Vesperini, E., D'Antona, F., McMillan, S. L. W., \& Recchi, S. 2008, MNRAS, 391, 825

Dobrovolskas, V., Kučinskas, A., Bonifacio, P., et al. 2014, A\& A, 565, A121

Georgy, C., Granada, A., Ekström, S., et al. 2014, A\& A, 566, A21

Girardi, L., Eggenberger, P., \& Miglio, A. 2011, MNRAS, 412, L103

Goudfrooij, P., Puzia, T. H., Chandar, R., \& Kozhurina-Platais, V. 2011, ApJ, 737, 4

Gratton, R. G., Carretta, E., \& Bragaglia, A. 2012, A\&SA Rev., 20, 50

Krause, M., Charbonnel, C., Decressin, T., Meynet, G., \& Prantzos, N. 2013, A\&A A, 552, A121

Krause, M., Charbonnel, C., Decressin, T., et al. 2012, A\&A, 546, L5

Larsen, S. S., Strader, J., \& Brodie, J. P. 2012, A\&A, 544, L14

Lind, K., Primas, F., Charbonnel, C., Grundahl, F., \& Asplund, M. 2009, A\&A, 503, 545

Mackey, A. D., Broby Nielsen, P., Ferguson, A. M. N., \& Richardson, J. C. 2008, ApJ, 681, L17

Maeder, A. \& Meynet, G. 2006, A\&A, 448, L37

Mengel, J. G. \& Gross, P. G. 1976, Ap\&SSS, 41, 407

Milone, A. P., Bedin, L. R., Piotto, G., \& Anderson, J. 2009, A\&A, 497, 755

Milone, A. P., Bedin, L. R., Piotto, G., et al. 2015, MNRAS, 450, 3750

Monaco, L., Villanova, S., Bonifacio, P., et al. 2012, A\&SA, 539, A157

Niederhofer, F., Georgy, C., Bastian, N., \& Ekström, S. 2015, MNRAS, 453, 2070

Prantzos, N., Charbonnel, C., \& Iliadis, C. 2007, A\& A, 470, 179

Rubele, S., Girardi, L., Kozhurina-Platais, V., et al. 2013, MNRAS, 430, 2774

Tailo, M., D'Antona, F., Vesperini, E., et al. 2015, Nature, 523, 318

Ulrich, R. K. 1972, ApJ, 172, 165

Ventura, P., D'Antona, F., Mazzitelli, I., \& Gratton, R. 2001, ApJ, 550, L65

Ventura, P., Di Criscienzo, M., Carini, R., \& D'Antona, F. 2013, MNRAS, 431, 3642

Zahn, J.-P. 1977, A\& A, 57, 383 